\title{
Novel Heterozygous Deletion in Retinol Dehydrogenase 12 (RDH12) Causes Familial Autosomal Dominant Retinitis Pigmentosa
}

\author{
Hajrah Sarkar', Adam M. Dubis ${ }^{1,2}$, Susan Downes ${ }^{3}$ and Mariya Moosajee 1,2,4* \\ ${ }^{1}$ Development, Ageing and Disease Theme, UCL Institute of Ophthalmology, London, United Kingdom, ${ }^{2}$ Department \\ of Genetics, Moorfields Eye Hospital NHS Foundation Trust, London, United Kingdom, ${ }^{3}$ Nuffield Department of Clinical \\ Neuroscience, University of Oxford, Oxford, United Kingdom, ${ }^{4}$ Department of Ophthalmology, Great Ormond Street Hospital \\ for Children NHS Foundation Trust, London, United Kingdom
}

\section{OPEN ACCESS}

Edited by:

Lawrence Todd Reiter,

The University of Tennessee Health

Science Center (UTHSC),

United States

Reviewed by:

Rui Chen,

Baylor College of Medicine,

United States

Abigail Fahim,

University of Michigan, United States

*Correspondence:

Mariya Moosajee

m.moosajee@ucl.ac.uk

Specialty section

This article was submitted to Genetic Disorders,

a section of the journal

Frontiers in Genetics

Received: 22 January 2020

Accepted: 20 March 2020

Published: 08 April 2020

Citation:

Sarkar H, Dubis AM, Downes S

and Moosajee M (2020) Novel

Heterozygous Deletion in Retinol Dehydrogenase 12 (RDH12) Causes

Familial Autosomal Dominant Retinitis

Pigmentosa. Front. Genet. 11:335.

doi: 10.3389/fgene.2020.00335
Mutations in the retinol dehydrogenase $12(R D H 12)$ gene are primarily associated with Leber congenital amaurosis (LCA) type 13, a severe early onset autosomal recessive retinal dystrophy. Only one family with a heterozygous variant, associated with mild retinitis pigmentosa (RP), has been reported. We report a novel heterozygous variant [(c.759del; p.(Phe254Leufs*24)], resulting in a frameshift and premature termination identified in two unrelated individuals with familial autosomal dominant RP. Both heterozygous variants are associated with a late onset RP phenotype, suggesting a possible genotype-phenotype correlation.

Keywords: RDH12, autosomal dominant, retinitis pigmentosa, adaptive optics, Leber congenital amaurosis

\section{INTRODUCTION}

Retinol dehydrogenase 12 (RDH12) is an NADPH-dependent retinal reductase that catalyzes the reduction of all-trans retinal to all-trans retinol, and is expressed in photoreceptor inner segments (Belyaeva et al., 2005). RDH12 has 7 coding exons, is located on chromosome $14 \mathrm{q} 24.1$ and encodes a 316 amino acid protein. Mutations in RDH12 are primarily associated with Leber congenital amaurosis (LCA), a severe early onset autosomal recessive retinal dystrophy, and accounts for 3.4-10.5\% of all cases (Kumaran et al., 2017). Patients present with signs of early onset central visual loss, nystagmus, not reaching or difficulty finding dropped objects and nyctalopia. This is a progressive disorder with significant decline from 10 years of age, which leads to complete blindness in adulthood. RDH12-LCA is characterized by macular atrophy, which extends peripherally in a variegated pattern corresponding to the retinal vasculature, and midperipheral pigmentary retinopathy (Aleman et al., 2018; Fahim et al., 2019). According to the Human Gene Mutation Database (HGMD), 80 unique RDH12 variants have been reported (HGMD public database accessed August 2019). One six-generation family with 19 affected members has been reported to harbor an autosomal dominant variant, c.776del; p.(Glu260Argfs*18), a single base pair deletion resulting in a frameshift 


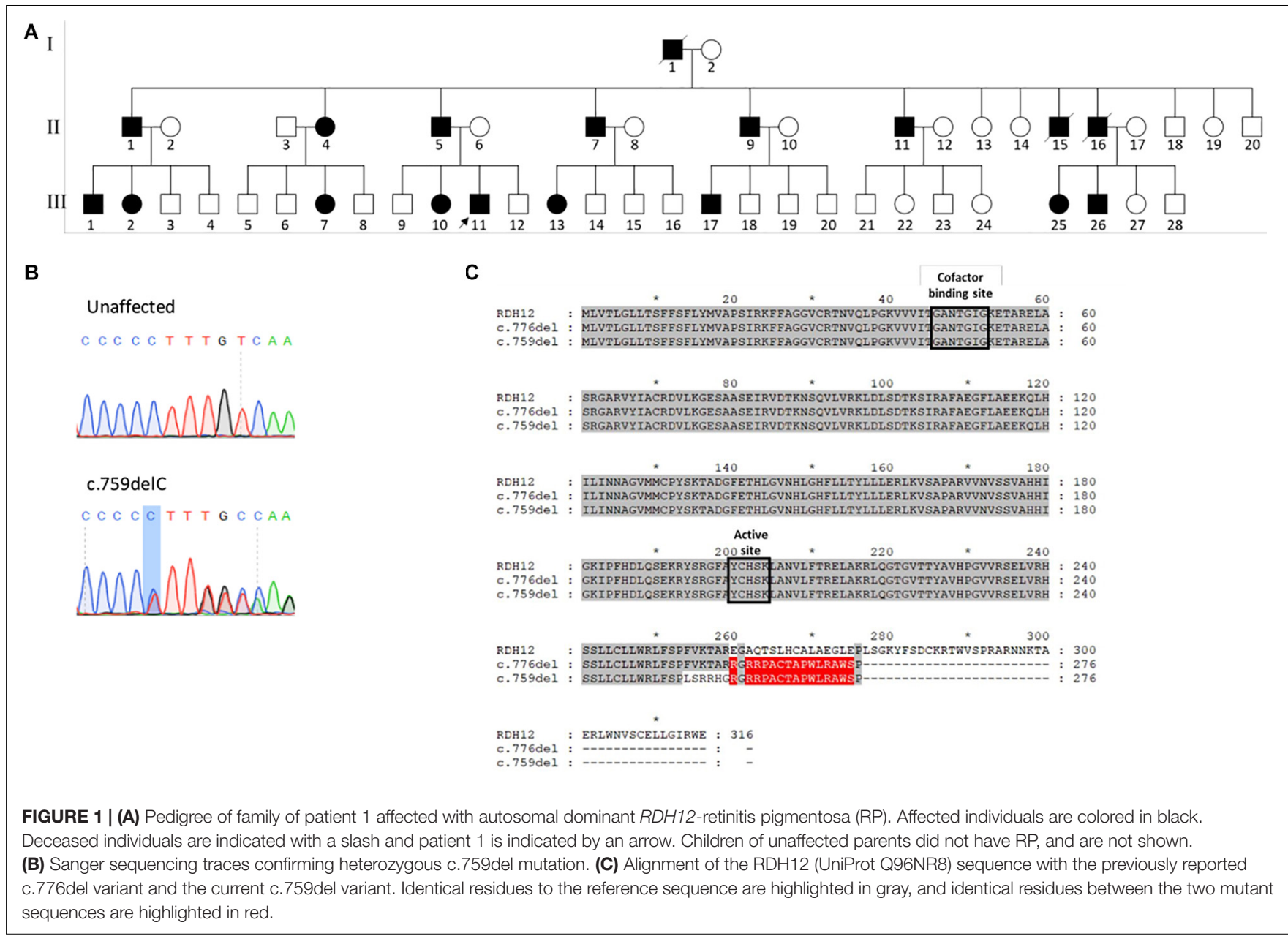

and premature termination. Affected individuals displayed a milder late onset (average age of diagnosis was 28.5 years) retinitis pigmentosa (RP) phenotype, with intraretinal bone spicule pigmentation and attenuation of retinal arterioles (Fingert et al., 2008). Herein this report, we describe two unrelated individuals with autosomal dominant RP associated with a novel point deletion in RDH12 with a similar phenotype. The patient study protocol adhered to the tenets of the Declaration of Helsinki, and received approval from the NRES Ethics Committee (REC12/LO/0141). Written informed consent was obtained from the individuals for the publication of any potentially identifiable images or data included in this article.

\section{CASE PRESENTATION}

Patient 1 is a 32-year-old Israeli man of mixed ethnicity, mother from Kurdistan and affected father from Tunisia, who presented with nyctalopia and field loss, but preserved central vision. He has an extensive positive family history exhibiting autosomal dominant inheritance with affected father, paternal grandfather and siblings (Figure 1A). His best corrected visual acuity with LogMAR was 0.04 in both eyes, fundus examination showed mild waxy disc pallor with retinal vessel attenuation and mid-peripheral bone-spicules. Dense spectral domain optical coherence tomography (SD-OCT) volumes were performed using the Heidelberg Spectralis (Heidelberg Engineering GmbH, Heidelberg, Germany). The area of SD-OCT is delineated by the yellow boxes on the fundus image in Figure 2A. The volume was acquired using high resolution, $1024 \mathrm{~A}$-scan/Bscan and $193 \mathrm{~B}$-scan over a $20 \times 20^{\circ}$ area. From this volume, the maximum intensity projection enface slab was generated (Chen et al., 2015) using the instrument software. Retinal layer segmentation was manually corrected for all B-scans by one observer (AMD). The slab was designed to measure the area of intact ellipsoid zone. This slab used the retinal pigment epithelium (RPE) contour and was defined as $10 \mu \mathrm{m}$ above the RPE with a thickness of $100 \mu \mathrm{m}$ (Figure 2C red lines). The maximum intensity slab showed an area of hyper-reflectivity surrounded by a ring of lower reflectance (dashed red line - Figure 2C). This pattern is due to the increased reflectivity from the intact ellipsoid zone band of the structural OCT and identifies the area of intact photoreceptors. This circular pattern is more reminiscent of RP than classic recessive RDH12 retinopathy (Aleman et al., 2018). Reflectance patterns can be compared to an age matched male control 
A
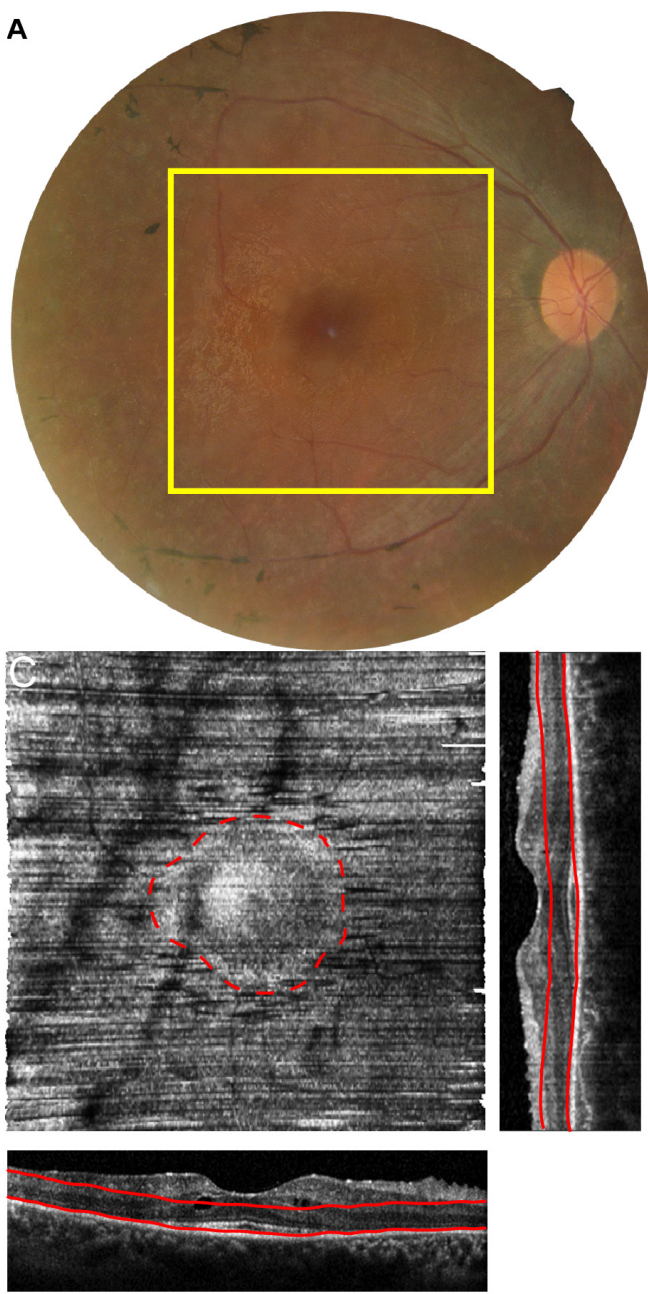

B
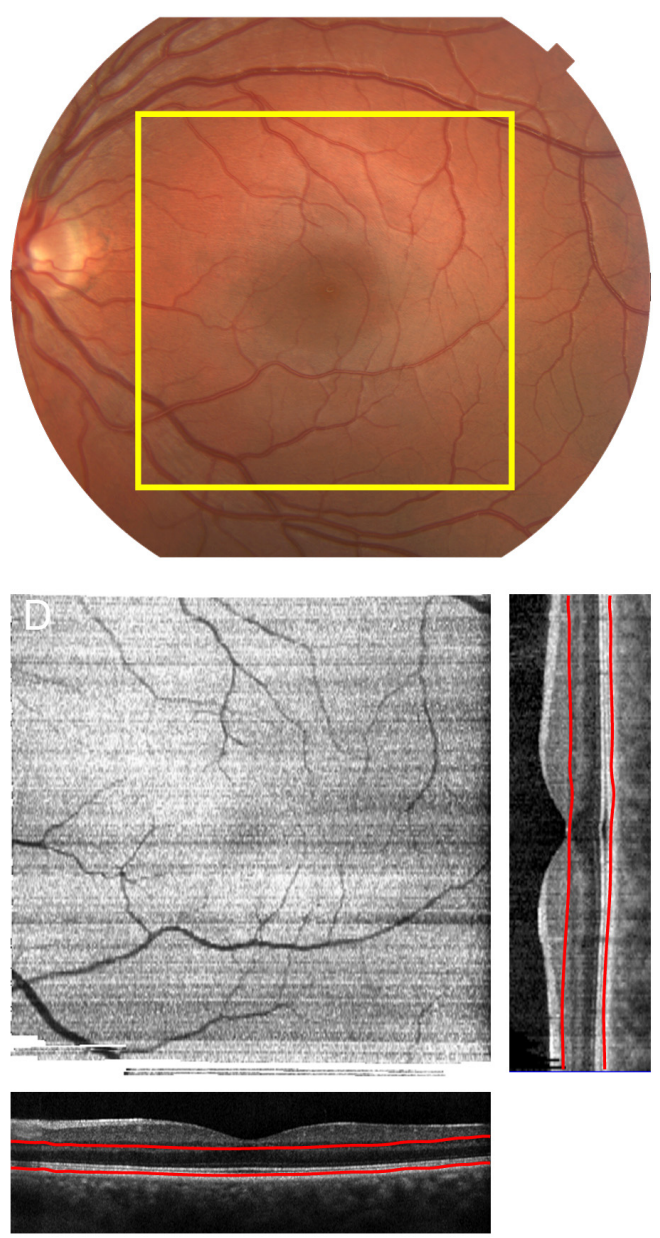

FIGURE 2 | Identification of Residual Photoreceptors through Enface OCT analysis. Macular SD-OCT images were acquired for a $20^{\circ} \times 20^{\circ}$ area in patient 1 (A) and age matched control (B). The RPE layer was segmented, with manual correction and a maximum intensity slab was generated for both subjects (C,D). The corresponding OCT, generated orthogonal OCTs are displayed on the side and bottom of each intensity projection. The red lines correspond to the location of the slab on the corresponding OCTs. The area of residual cones is highlighted in C (red dashed line).

(Figures 2B,D). In this subject the maximum intensity projection slab, using the same bounding as the patient, shows a uniform reflectance characteristic of having an intact ellipsoid zone over the entire region.

There was salient hypo- and hyper-autofluorescence rings present on short-wavelength fundus autofluorescence (FAF) and residual foveal island on SD-OCT (Figure 3). Parafoveal macular edema was present in both eyes. Adaptive optics scanning laser ophthalmoscopy (AOSLO) using a bespoke system with multidetector schemes was performed on both eyes (Dubra et al., 2011; Scoles et al., 2014). Foveal cone morphology and density was relatively normal (Figure 3 ). Cone density remained relatively preserved across the residual OCT ellipsoid zone (EZ) island, confirmed by cellular structures present in the split detection images. However, confocal imaging showed a drastic reduction in normal appearing foveal cones (bright Gaussian profile spots), even in areas without visible macular edema (Figure 3). Split detection imaging also suggested some cellular structures outside the EZ island. Intact structures can be inferred from the round, dimple-like structures present in the split detection view.

The patient underwent whole exome sequencing and was found to have a heterozygous deletion in RDH12 (NM_152443.2), c.759del; p.(Phe254Leufs*24), which segregated with his affected father and sister, and was confirmed by Sanger sequencing (Figure 1B). This variant has not been reported on gnomAD. The pathogenicity of the variant was scored with SIFT_Indel (Hu and Ng, 2013), Variant Effect Predictor (McLaren et al., 2016) and MutationTaster (Schwarz et al., 2014), and all classified the variant as damaging or disease causing. No disease causing mutations were found in any other known retinal disease genes.

Patient 2 is an unrelated 37-year-old Caucasian English man with a maternal family history of RP with an affected mother and maternal grandfather. He had a history of keratoconus from adolescence, and at age 24 has an episode of left hydrops 


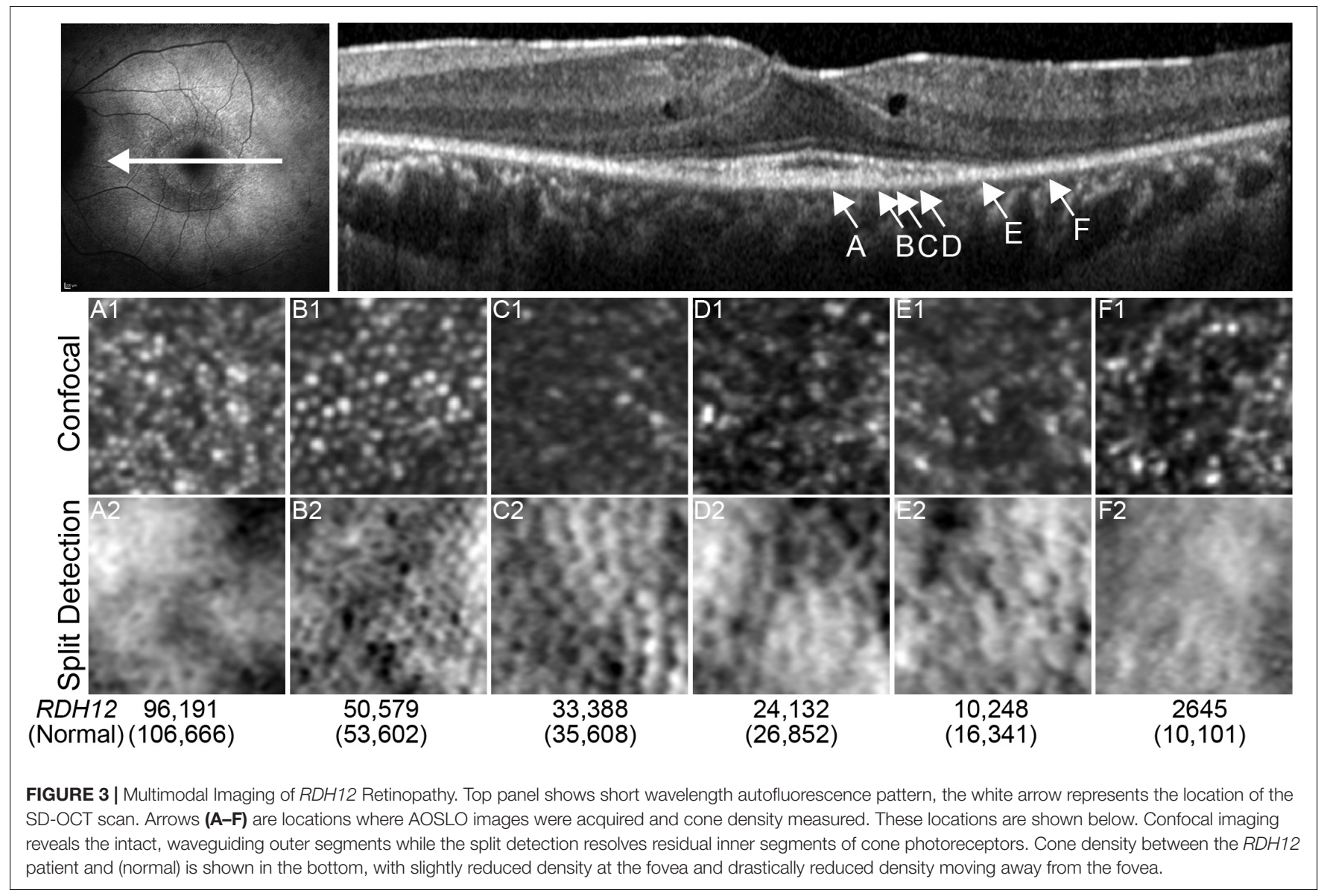

which required a penetrating keratoplasty. After this he began to notice nyctalopia and progressive visual field loss. He maintains good central and color vision. His best corrected visual acuity is 0.18 LogMAR in both eyes and $17 / 17$ color vision using Ishihara. Fundus examination showed mild waxy disc pallor with retinal vessel attenuation and mid-peripheral bone-spicules similar to patient 1 (Figure 4). Ultra-widefield retinal autofluorescence imaging displayed the characteristic hypo- and hyper-autofluorescence rings around the residual foveal island. Whole exome sequencing revealed the same heterozygous variant as patient 1 , which segregated with his affected mother.

The mutation is located toward the end of the reading frame, with $80 \%$ of the sequence unchanged, including the two highly conserved catalytic domains: the cofactor binding site at positions 46-52, and the active site at 200204, possibly accounting for the relatively mild phenotype (Figure 1C). This mutation results in loss of the terminal 63 amino acids, which are highly conserved across species, causing premature termination at codon 277. Due to the proximity of the mutation to the final exon-exon junction, this transcript is likely to escape nonsense mediated decay (NMD) and result in the expression of a truncated protein. This variant is only $17 \mathrm{bp}$ upstream from the previously reported heterozygous variant, and both give rise to a 277 amino acid protein, with a common 17 amino acid C-terminal sequence (Figure 1C).

\section{DISCUSSION}

Here, we describe two unrelated families with a novel autosomal dominant variant in RDH12, displaying a RP phenotype (Gill et al., 2019). In patients with autosomal recessive $R D H 12$ variants, Aleman et al. (2018) described a rapidly progressing condition leaving only residual islands intact by the second decade of life with very poor visual acuity. The cases presented here are in stark contrast to this classical representation with relatively intact macular structure and visual acuity. The comparison is further made by the presence of the classic $\mathrm{RP}$ autofluorescent ring pattern compared to the much more variegated pattern in previous $\mathrm{RDH} 12$ reports (Kumaran et al., 2017; Aleman et al., 2018).

Eighty RDH12 mutations have been reported to date, $64 \%$ of which are missense, $15 \%$ are nonsense and $14 \%$ are small insertions or deletions, including 6 autosomal recessive deletions associated with the severe early onset LCA phenotype (HGMD public database accessed August 2019). A 5bp deletion in the terminal exon, c.806_810del; Ala269Glyfs*2, has been reported in patients with autosomal 
A
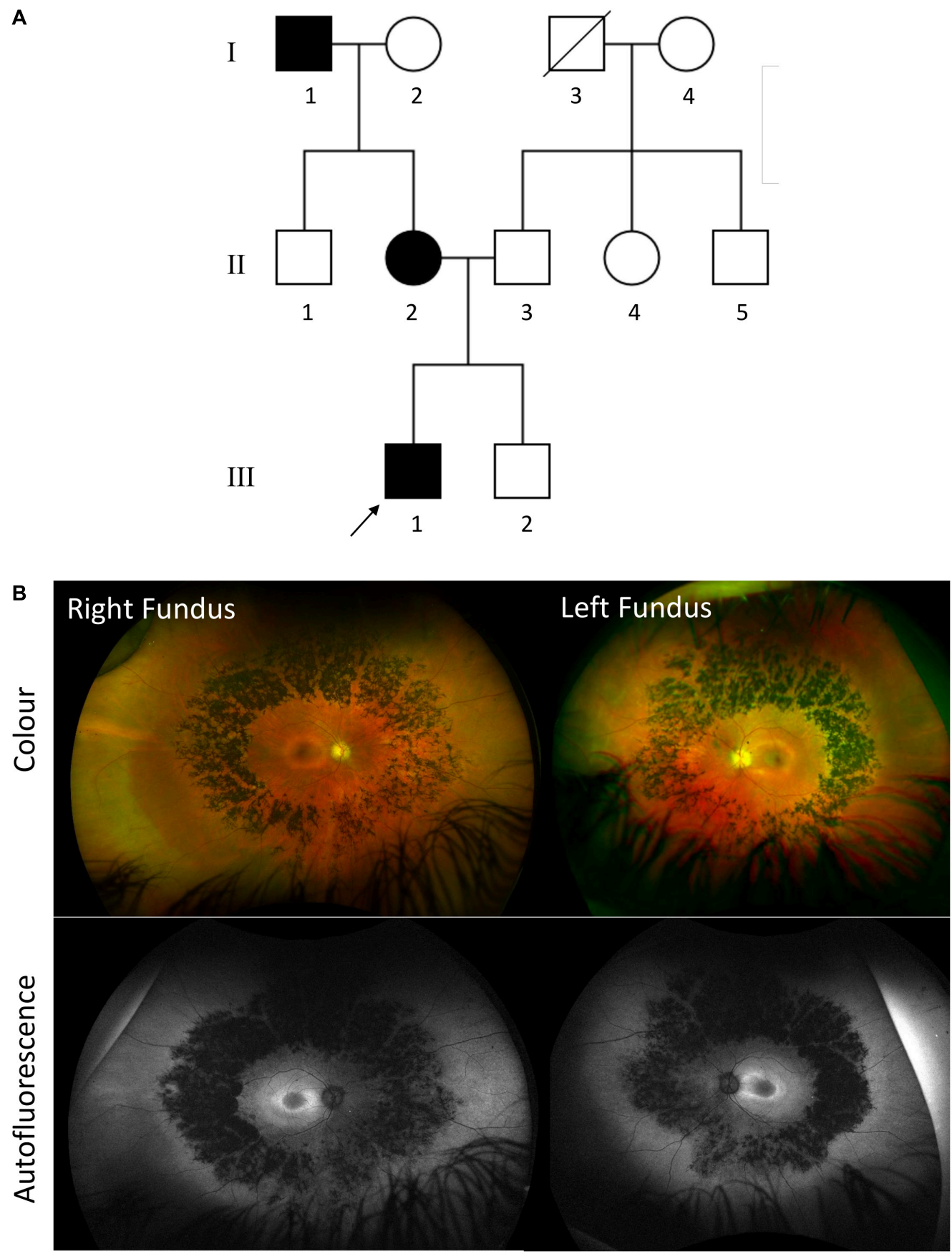

FIGURE 4 | (A) Family pedigree of patient 2 affected with autosomal dominant RDH12-retinitis pigmentosa (RP). Affected individuals are colored in black. Deceased individuals are indicated with a slash and patient 2 is indicated by an arrow. (B) Ultrawidefield Optos color images of right and left fundus of patient 2 with corresponding autofluorescence.

recessive LCA, which was shown to result in loss of function, with less than $5 \%$ enzyme activity seen in vitro (Sun et al., 2007). This variant results in a truncated protein with only two out-of-frame amino acids, as opposed to the identical 17 out-of-frame amino acids seen in both dominant variants. This C-terminal peptide sequence produced by the dominant variants may be responsible for a protein interaction resulting in gain of function or a dominant negative effect, which may account for the milder phenotype. However, further functional studies into the differences in disease mechanisms between autosomal recessive and autosomal dominant mutations are required. 


\section{DATA AVAILABILITY STATEMENT}

The datasets generated for this study can be found in ClinVar (SCV001164601).

\section{ETHICS STATEMENT}

Written informed consent was obtained from the individuals for the publication of any potentially identifiable images or data included in this article.

\section{AUTHOR CONTRIBUTIONS}

HS performed the experiments for Sanger sequencing validation of mutation. AD performed ophthalmic imaging of the patients.

\section{REFERENCES}

Aleman, T. S., Uyhazi, K. E., Serrano, L. W., Vasireddy, V., Bowman, S. J., Ammar, M. J., et al. (2018). RDH12 mutations cause a severe retinal degeneration with relatively spared rod function. Invest. Ophthalmol. Vis. Sci. 59, 5225-5236. doi: $10.1167 /$ iovs. $18-24708$

Belyaeva, O. V., Korkina, O. V., Stetsenko, A. V., Kim, T., Nelson, P. S., and Kedishvili, N. Y. (2005). Biochemical properties of purified human retinol dehydrogenase 12 (RDH12): catalytic efficiency toward retinoids and C9 aldehydes and effects of cellular retinol-binding protein type I (CRBPI) and cellular retinaldehyde-binding protein (CRALBP) on the oxidation and reduction of retinoids. Biochemistry 44, 7035-7047. doi: 10.1021/bi050 $226 \mathrm{k}$

Chen, Q., Niu, S., Shen, H., Leng, T., de Sisternes, L., and Rubin, D. L. (2015). Restricted Summed-Area Projection for Geographic Atrophy Visualization in SD-OCT Images. Transl Vis Sci Technol 4:2. doi: 10.1167/tvst.4.5.2

Dubra, A., Sulai, Y., Norris, J. L., Cooper, R. F., Dubis, A. M., Williams, D. R., et al. (2011). Noninvasive imaging of the human rod photoreceptor mosaic using a confocal adaptive optics scanning ophthalmoscope. Biomed. Opt. Express 2, 1864-1876. doi: 10.1364/boe.2.001864

Fahim, A. T., Bouzia, Z., Branham, K. H., Kumaran, N., Vargas, M. E., Feathers, K. L., et al. (2019). Detailed clinical characterisation, unique features and natural history of autosomal recessive $\mathrm{RDH} 12$-associated retinal degeneration. $\mathrm{Br}$. J. Ophthalmol. 103, 1789-1796. doi: 10.1136/bjophthalmol-2018-313580

Fingert, J. H., Oh, K., Chung, M., Scheetz, T. E., Andorf, J. L., Johnson, R. M., et al. (2008). Association of a novel mutation in the retinol dehydrogenase 12 (RDH12) gene with autosomal dominant retinitis pigmentosa. Arch. Ophthalmol. 126, 1301-1307. doi: 10.1001/archopht.126.9.1301

Gill, J. S., Moosajee, M., and Dubis, A. M. (2019). Cellular imaging of inherited retinal diseases using adaptive optics. Eye 33, 1683-1698. doi: 10.1038/s41433019-0474-473
MM and SD conducted the clinical evaluation of the patients. HS, $\mathrm{AD}$, and $\mathrm{MM}$ wrote the manuscript.

\section{FUNDING}

This work was funded by Retina UK, the NIHR Biomedical Resource Centre at Moorfields Eye Hospital NHS Trust and UCL Institute of Ophthalmology and Wellcome Trust (Grant Number 205174/Z/16/Z).

\section{ACKNOWLEDGMENTS}

The authors would like to thank Alfredo Dubra for sharing AOSLO acquisition and image registration software.

$\mathrm{Hu}, \mathrm{J}$, and Ng, P. C. (2013). SIFT Indel: predictions for the functional effects of amino acid insertions/deletions in proteins. PLoS One 8:e77940. doi: 10.1371/ journal.pone.0077940

Kumaran, N., Moore, A. T., Weleber, R. G., and Michaelides, M. (2017). Leber congenital amaurosis/early-onset severe retinal dystrophy: clinical features, molecular genetics and therapeutic interventions. Br. J. Ophthalmol. 101, 11471154. doi: 10.1136/bjophthalmol-2016-309975

McLaren, W., Gil, L., Hunt, S. E., Riat, H. S., Ritchie, G. R., Thormann, A., et al. (2016). The ensembl variant effect predictor. Genome Biol. 17:122. doi: 10.1186/ s13059-016-0974-974

Schwarz, J. M., Cooper, D. N., Schuelke, M., and Seelow, D. (2014). MutationTaster2: mutation prediction for the deep-sequencing age. Nat. Methods 11, 361-362. doi: 10.1038/nmeth.2890

Scoles, D., Sulai, Y. N., Langlo, C. S., Fishman, G. A., Curcio, C. A., Carroll, J., et al. (2014). In vivo imaging of human cone photoreceptor inner segments. Invest. Ophthalmol. Vis. Sci. 55, 4244-4251. doi: 10.1167/iovs.14-14542

Sun, W., Gerth, C., Maeda, A., Lodowski, D. T., Van Der Kraak, L., Saperstein, D. A., et al. (2007). Novel RDH12 mutations associated with Leber congenital amaurosis and cone-rod dystrophy: biochemical and clinical evaluations. Vis. Res. 47, 2055-2066. doi: 10.1016/j.visres.2007.04.005

Conflict of Interest: The authors declare that the research was conducted in the absence of any commercial or financial relationships that could be construed as a potential conflict of interest.

Copyright (c) 2020 Sarkar, Dubis, Downes and Moosajee. This is an open-access article distributed under the terms of the Creative Commons Attribution License (CC BY). The use, distribution or reproduction in other forums is permitted, provided the original author(s) and the copyright owner(s) are credited and that the original publication in this journal is cited, in accordance with accepted academic practice. No use, distribution or reproduction is permitted which does not comply with these terms. 